November 28, 2017 16:47 WSPC/INSTRUCTION FILE 390IsoCosmFINAL140418

International Journal of Geometric Methods in Modern Physics

(C) World Scientific Publishing Company

\title{
ISOCHRONOUS COSMOLOGIES
}

\author{
FABIO BRISCESE \\ Istituto Nazionale di Alta Matematica Francesco Severi, Gruppo Nazionale di Fisica \\ Matematica, Città Universitaria, P.le A. Moro 5, 00185 Rome, Italy. \\ and \\ Dipartimento SBAI, Sezione di Matematica, Sapienza Università di Roma, Via Antonio Scarpa \\ 16, 00161 Rome, Italy. \\ fabio.briscese@sbai.uniroma1.it \\ FRANCESCO CALOGERO \\ Dipartimento di Fisica, Università di Roma "La Sapienza", Rome, Italy. \\ and \\ Istituto Nazionale di Fisica Nucleare, Sezione di Roma, Rome, Italy. \\ francesco.calogero@roma1.infn.it,francesco.calogero@uniroma1.it
}

Received (20 January 2014)

Revised (04 February 2014)

\begin{abstract}
The possibility has been recently demonstrated to manufacture (nonrelativistic, Hamiltonian) many-body problems which feature an isochronous time evolution with an arbitrarily assigned period $T$ yet mimic with good approximation, or even exactly, any given many-body problem (within a large, physically relevant, class) over times $\tilde{T}$ which may also be arbitrarily large (but of course such that $\tilde{T}<T$ ). Purpose and scope of this paper is to explore the possibility to extend this finding to a general relativity context. For simplicity we restrict our consideration to the case of homogeneous and isotropic metrics and show that, via an approach analogous to that used for the nonrelativistic many-body problem, a class of homogeneous and isotropic cyclic solutions of Einstein's equations may be obtained. For these solutions the duration of the cycles does not depend on the initial conditions, so we call these models isochronous cosmologies. We give a physical interpretation of such metrics and in particular we show that they may behave arbitrarily closely, or even identically, to the Friedman-Robertson-Walker solutions of Einstein's equations for an arbitrarily long time (of course shorter than their period, which can also be assigned arbitrarily), so that they may reproduce all the satisfactory phenomenological features of the standard cosmological $\Lambda$-CDM model in a portion of their cycle; while these isochronous cosmologies may be geodesically complete and therefore singularity-free.
\end{abstract}

Keywords: Cosmology; Isochronous Systems; General Relativity.

\section{Introduction}

It has been recently shown 12 how, given a quite general (autonomous) dynamical system $D$, other (also autonomous) dynamical systems $\tilde{D}$ can be manufactured, featuring two additional arbitrary positive parameters $T$ and $\tilde{T}$ with $T>\tilde{T}$ (and 
possibly also two additional dynamical variables) and having the following two properties: (i) For the same variables of the original dynamical system $D$ the new dynamical system $\tilde{D}$ yields, over the time interval $\tilde{T}$, hence for an arbitrarily long time, a dynamical evolution which mimics arbitrarily closely that yielded by the original system $D$; up to corrections of order $t / \tilde{T}$, or possibly even identically. (ii) The system $\tilde{D}$ is isochronous: all its solutions (for arbitrary initial data) are completely periodic with the assigned period $T$. A particularly interesting example of this phenomenon is the standard Hamiltonian system describing, in an ambient space of arbitrary dimensions $d$ (including of course $d=3$ ), an arbitrary number $N$ of point particles with arbitrary masses, interacting among themselves via potentials depending arbitrarily from the particle coordinates. Moreover it has been shown [12] that, if this (autonomous) Hamiltonian $H$ is translation-invariant (i. e., it features no external potentials), new (also autonomous) Hamiltonians $\tilde{H}$ characterizing modified many-body problems can be manufactured that feature the same dynamical variables as $H$ (i. e., there is then no need to introduce two additional dynamical variables) and yield a time evolution quite close, or even identical to that yielded by the original Hamiltonian $H$ over the arbitrarily assigned time $\tilde{T}$, while being isochronous with the arbitrarily assigned period $T$ (of course with $T>\tilde{T}$ ).

The Hamiltonian model $H$ described above clearly encompasses a lot of physics; and since it is difficult, or perhaps impossible, to distinguish experimentally dynamical systems that behave arbitrarily closely, or even identically, over an arbitrarily long period of time, this finding - which is a proven mathematical theorem, at least for realistic Hamiltonian many-body problems predicting a nonsingular future, as it is natural in physics - has various remarkable implications for all those who believe that physics is a science based on experimental verifications of its laws. In particular it raises [12] interesting questions about the distinction between integrable and nonintegrable evolutions, the definition of chaotic behavior, the applicability of statistical mechanics and the validity of the second principle of thermodynamics (say, for $N \approx 10^{24}$ ), and about cosmology (say, for $N \approx 10^{85}$; including, in this case, issues which have an eschatological connotation reminiscent of "eternal return" conceptions which are, however, not our cup of tea).

The present paper is motivated by the potential relevance of the findings outlined above for general relativity. Can some analogous finding be obtained concerning cosmology, but in the context of general relativity rather than classical (nonrelativistic) mechanics?

Remark 1.1. Actually the finding described above can also be extended to a quantum mechanical context, up to the ambiguities inherent in the transition from classical to quantum mechanics (particularly significant for Hamiltonians with a nonconventional kinetic energy component, as featured by the modified Hamiltonians mentioned above) [1]. Anyway in this paper we refrain from considering any quantum-mechanical context, which would anyway be impossible as long as no framework is available encompassing in a mathematically rigorous fashion both 
general relativity and quantum theory.

Although the results outlined above are valid for autonomous systems, they are in fact based on the introduction of an auxiliary variable in place of the physical time variable 2. In particular the dynamical systems $D$ and $\tilde{D}$ mentioned above feature the same trajectories in phase space, but while the time evolution of the dynamical system $D$ corresponds to a uniform forward motion along those trajectories, the time evolutions of the modified dynamical systems $\tilde{D}$-although produced by timeindependent equations of motion - correspond to a periodic (with assigned period $T$ ), forward and backward, time evolution along those same trajectories, exploring of course only a portion of them; with the possibility that, for a subinterval $\tilde{T}<T$, this motion be also uniform, entailing that on such subinterval the dynamics of $D$ and $\tilde{D}$ are indeed identical. Let us emphasize that, while this procedure entails the introduction of an auxiliary variable $\tau \equiv \tau(t)$, appropriately related to the physical time $t$, any attempt to attribute to this new variable $\tau$ the significance of "time" would be completely unjustified: quite improper and confusing.

In classical mechanics, these different behaviors are produced by different (autonomous) Hamiltonians (some of which, as indicated above, may however be hardly distinguishable by experiments involving an arbitrarily long, but finite, time evolution). We show below that, in the context of general relativity, an analogous phenomenology also exists, which however does not require any change of the fundamental Einstein equations, corresponding instead to the identification of an enlarged class of metrics associated to the solutions of these equations. This shall again entail the introduction of a new auxiliary variable $\tau \equiv \tau(t)$-where $t$ is the physical time, itself defined up to diffeomorphic transformations as implied by the general relativity context; and again any attempt to attribute globally to this auxiliary variable $\tau$ the significance of time would be completely unjustified, improper and confusing.

One last caveat before embarking in the detailed presentation of our results. The previous findings concerning, in a nonrelativistic context, the general many-body problem [1/2] were not meant to suggest that the modified Hamiltonians yielding isochronous motions are more appropriate than the standard Hamiltonians as descriptions of any specific many-body problem - including that involving a number of particles comparable to those of the entire universe (assuming this number has any sense). It was merely meant to demonstrate a, perhaps unpleasant but unfortunately inescapable, feature of any attempt to describe a physical many-body problem in the context of Hamiltonian dynamics: the fact that different Hamiltonians exist which characterize different physical behaviors which may however be difficult, in fact impossible, to distinguish experimentally. Of course this fact could be simply ignored, for one's peace of mind; albeit at the risk of eventually discovering that the pursuit of the peace of one's mind is not the proper approach to the advancement of science (indeed, how to exclude altogether the - presumably very unlikely, but not physically forbidden - possibility that one of the modified Hamiltonians turn out to describe reality better than the standard one?). Likewise the 
present paper-which is focussed on cosmology and in that context shows that the framework of general relativity includes a larger class of cosmological solutions than usually considered and that these solutions may describe isochronous universes - is not meant to argue that our Universe does evolve isochronously; but merely that such possibilities are contained in the equations of general relativity-compatibly with what is observationally known about our Universe - and might indeed have some interesting connotations, for instance avoid Big Bang singularities.

For simplicity in this paper we focus on cosmological solutions of the Einstein equations providing a highly idealized (homogeneous and isotropic) picture of the universe; which is indeed the standard point of departure of cosmological investigations (see for instance [3]). Applying techniques analogous to those outlined above [12], we obtain isochronous (homogeneous and isotropic) cosmological solutions of Einstein's equations and tersely analyze their properties.

Periodic cosmological solutions of Einstein's equations have already been abundantly discussed as alternatives to the standard cosmological $\Lambda$-CDM model consisting (see for instance [3]) of a Friedmann-Robertson-Walker (FRW) homogeneous and isotropic universe filled with standard particles plus a cosmological constant $\Lambda$ plus some amount of dark matter. Let us recall in this connection that - in spite of the fact that the $\Lambda$-CDM model is rather successful at explaining the data $4|5| 6 / 7 / 8|9| 10 \mid 11 / 213$ - some questions remain open. For example the the appropriate value of the cosmological constant cannot be deduced from a fundamental theory, due to a severe fine tuning problem. And it is unclear which inflaton field should be associated to the primordial inflation. Moreover the $\Lambda$-CDM model features a Big Bang singularity and requires a fine tuning between the energy density of matter, radiation and dark energy as well as specific initial conditions.

An example of cyclic universe makes use of a scalar field with a specific potential such that the universe starts from a big bang and ends with a big crunch [14]. Other examples are the ekpyrotic scenario [15] based on brane theory, the string theory inspired bouncing cosmologies [16] and the periodic cosmologies based on Chaplygin gas [17. All these models make use of some string theory inspired scalar or vector field, or of a perfect fluid with a nonlinear equation of state (see [18 for a review), resulting in a FRW universe filled with some exotic fluid.

In the present paper we extend to general relativity the results on isochronous systems valid in the context of nonrelativistic Hamiltonian many-body problems. We thus obtain a class of cyclic solutions of Einstein's equations which are homogeneous and isotropic in space. These solutions are cyclic even if they contain no exotic fluids but only ideal fluids. Since the duration of each cycle does not depend on initial conditions we call them isochronous cosmological solutions. Another remarkable property of these solutions is that they can mimic the FRW solutions of Einstein's equations accurately for an arbitrarily long time. Therefore they are no less in agreement with cosmological data than the $\Lambda$-CDM model, giving the right sequence of inflation / radiation domination / matter domination / late time acceleration epochs. Moreover, they may be free of singularities: the big bang / 
big crunch singularities may be avoided because in these solutions the contraction and expansion of the universe may be reversed at some finite (neither vanishing nor diverging) value of the scale factor. Therefore there is no need to explain the transition from a big crunch to a big bang as is the case for other cyclic universes 14.

Let us conclude this preliminary section by noting that the solutions of Einstein's equations introduced in this paper entail metrics that are considered unacceptable by some colleagues: indeed we experienced difficulties in having this paper published, because some referees ruled out these solutions as unphysical. This opinion was generally based on the unjustified interpretation of the quantity $\tau \equiv \tau(t)$ (see below) as a "global time", rather than an auxiliary function of the time $t$ (as in the nonrelativistic case described above and treated in [2]). In fact, as shown below, the only peculiarity of the metrics we consider is that they are degenerate at some discrete values of the parameter $t$ which - up to diffeomorphic reparameterizationhas in our models the significance of "time"; a feature that is also shared by other standard solutions of Einstein's equations which are nevertheless generally considered quite acceptable, such as, for instance, the well-known Schwarzschild solution. The arguments of these colleagues seemed to us as unyielding to our efforts to clarify matters as Cesare Cremonini's refusal to look into Galileo's telescope [19. We hope that the publication of this paper will allow the scientific community to judge whether this extension of the class of solutions of Einstein's equations should be considered acceptable and the corresponding physical arguments worthy of serious consideration (incidentally, we will be happy to share with any interested reader our previous exchanges with referees and editors). Let us reiterate that we are not asserting in this paper that the class of solutions we introduce provide the correct (of course approximate) description of the Universe we live in; but we submit that they should be taken into proper consideration, as they seem to us to feature - as indicated above and below - certain, possibly more appealing, aspects than alternative descriptions in the framework of general relativity of an (isotropic and homogeneous) isochronous Universe. In any case it seems to us that one should not ignore the possibility to construct - as shown below - for any given solution of the Einstein equations, another solution - in fact, an infinity of such solutions - which are physically essentially indistinguishable from the given one over some (arbitrarily long) time interval but are periodic on a longer time scale.

\section{Isochronous Cosmologies}

Our point of departure is to consider, in a given reference frame $(t, \vec{x})$, an homogeneous and isotropic metric reading

$$
d s^{2}=b(t)^{2} d t^{2}-a(t)^{2} d \vec{x}^{2}
$$

with $b(t)$ a periodic function (with an arbitrarily assigned period $T$ ) having moreover a vanishing mean value, so that its integral $B(t)$ is also periodic with period 
$T:$

$$
b(t+T)=b(t) ; \quad B(t) \equiv \int_{0}^{t} b\left(t^{\prime}\right) d t^{\prime}, \quad B(t+T)=B(t) .
$$

Of course this metric can be mapped locally into the FRW metric

$$
d s^{2}=d \tau^{2}-\alpha(\tau)^{2} d \vec{x}^{2}, \quad \alpha(\tau) \equiv a(t),
$$

via the change of variable

$$
d \tau=b(t) d t, \quad \tau(t)=B(t) .
$$

Hence one might infer that the new metric (1) is physically equivalent to the FRW metric (2), since in general relativity different metrics related by a reparameterization of the coordinates - and in particular of the time variable - are considered physically equivalent. But this is the case only if the relevant reparameterization is univocally invertible (technically, a diffeomorphism). This is indeed the case for the change of variables (2b) locally, but not globally; in particular, certainly not over a time interval equal to or larger than $T$, because obviously the periodic functions $b(t)$ and $B(t)$ are certainly not univocally invertible over time intervals equal to or larger than $T$ (see (1b); in fact, here and below $T$ should be replaced by $T / 2$ for the assignment (9), see below). Hence the two metrics (1) and (2) are not physically equivalent: they represent two physically different solutions of the Einstein equations. Let us reemphasize this point: the metric (11) is not globally reducible via a diffeomorphism to the FRW metric (2) hence the class of solutions of the Einstein's equations characterized by this metric (11) represent a physically different universe from those characterized by the FRW metric (2). In a given spacetime described by the metric (11) an observer is allowed to change the way time is measured-i. e., to perform a diffeomorphic transformation of the time variable - in order to reduce the spacetime metric to the FRW metric (2); but only for an interval of time $\tilde{T}$ sufficiently smaller than $T$. Over any time interval larger than $T$ - or even insufficiently smaller than $T$, see below - a departure from the FRW metric (2) shall necessarily emerge. Indeed we show below that the new solutions associated to the metric (10) are physically different from those associated to the metric (22): for instance in the first case - in contrast to the second - there might be no Big Bang (see below). And we also indicate why there is no justification to exclude a priori these solutions.

As usual in cosmological investigations based on the Einstein equations, we assume that the energy-momentum tensor of the universe is that of an ideal fluid with a 4-velocity given by $U_{\mu}=|b(t)| \delta_{\mu 0}$ (the extension to more general energymomentum tensors is trivial), so that the ideal fluid has zero spatial velocity and the frame $(t, \vec{x})$ is co-moving, while the zero component of the 4-velocity is always nonnegative (as it should be). The Einstein equations for the metric (11) are then drastically simplified [3]. The corresponding generalized FRW equations read

$$
3\left[\frac{\dot{a}(t)}{a(t)}\right]^{2}=k b(t)^{2} \rho(t),
$$




$$
\dot{\rho}(t)+3\left[\frac{\dot{a}(t)}{a(t)}\right][\rho(t)+P(t)]=0 .
$$

Here and hereafter superimposed dots denot differentiation with respect to the time $t ; \rho$ and $P$ are respectively the energy density and pressure of the universe; $k=8 \pi G / c^{4} ; G$ is the gravitational constant; $c$ the speed of light. We stress that these equations are obtained in the framework of Einstein's theory and they do not assume any modification of general relativity.

This system of two ODEs must be complemented by an equation of state relating the pressure $P$ of the universe to its energy density $\rho$ : we shall here use for simplicity the simple ideal fluid relation $P=\omega \rho$, with constant $\omega$. But note that, after this assignment, the system (3) is not quite closed: it is still possible to assign, essentially arbitrarily, the function $b(t)$. Of course this arbitrariness corresponds to the freedom in general relativity to reparametrize time; but - as discussed above - it goes beyond this if $b(t)$ is not invertible, hence if the relevant reparameterization is not a global diffeomorphism.

If we consider a perfect fluid with constant equation of state parameter $\omega>-1$, it follows from (3b) that the energy density of the fluid is

$$
\rho(t)=\rho(0)\left[\frac{a(0)}{a(t)}\right]^{3(\omega+1)}, \quad \text { if } \quad \omega>-1,
$$

while for $\omega=-1$ one has a constant energy density,

$$
\rho=\Lambda \quad \text { if } \quad \omega=-1
$$

It is now convenient [2] to introduce a new variable $\tau$ by setting (see (1b )

$$
\tau(t) \equiv B(t)
$$

and new auxiliary functions $\alpha(\tau), r(\tau), p(\tau)$ by setting

$$
a(t) \equiv \alpha(\tau(t)), \quad \rho(t) \equiv r(\tau(t)), \quad P(t) \equiv p(\tau(t))
$$

Then the functions $a(t)$ and $\rho(t)$ are solutions of (3) provided $\alpha(\tau)$ and $r(\tau)$ are solutions of the following system:

$$
\begin{gathered}
3\left[\frac{\alpha^{\prime}(\tau)}{\alpha(\tau)}\right]^{2}=k r(\tau), \\
r^{\prime}(\tau)+3\left[\frac{\alpha^{\prime}(\tau)}{\alpha(\tau)}\right][r(\tau)+p(\tau)]=0 .
\end{gathered}
$$

Here the prime denotes differentiation with respect to $\tau$, and $p(\tau)$ and $r(\tau)$ satisfy the same equation of state $p(\tau)=\omega r(\tau)$ as $P(t)$ and $\rho(t)$. And since the definition of $\tau(t)$ (see (6a) with (1b) ) implies that this function is periodic with period $T$,

$$
\tau(t+T)=\tau(t)
$$


the same property is inherited via the definitions (6b) by the physical quantities $a(t), \rho(t)$ and $P(t)$ :

$$
a(t+T)=a(t), \quad \rho(t+T)=\rho(t), \quad P(t+T)=P(t) .
$$

Note that this property holds now for the solutions of (3) characterized by any initial conditions: the physical quantities $a(t), \rho(t)$ and $P(t)$ are therefore isochronous. Moreover, since $b(t)$ can be assigned arbitrarily, the period $T$ of these isochronous solutions is a parameter which can be freely assigned.

But let us re-emphasize that the variable $\tau$ cannot be given globally the significance of "time", hence the fact that $\dot{\tau}$ might change sign - indeed, it certainly does so, see (6a) and (1b) - has no unphysical connotation.

It is immediate to identify (7) with the FRW system characterized by the metric (2a) with the energy momentum tensor of a perfect fluid in co-moving coordinates. Therefore (6) says that the solutions $a(\tau), \rho(\tau), p(\tau)$ of the FRW system (7) are locally mapped into the solutions of (3) via the local time reparameterization (6). But, as emphasized above, due to the periodicity of $\tau(t)$, this mapping cannot be globally extended since the change of variable (6) is not a diffeomorphism for all $t$, therefore $a(t), \rho(t), P(t)$ and $\alpha(\tau), r(\tau), p(\tau)$ correspond in fact to different cosmologies.

Below we present two explicit examples of isochronous cosmological solutions of Einstein's equations obtained in this manner and describe their main properties. In both cases we assign for simplicity the function $b(t)$ and $B(t)$ as follows:

$$
b(t) \equiv \cos (\Omega t), \quad B(t)=\frac{\sin (\Omega t)}{\Omega}, \quad \Omega=\frac{2 \pi}{T} .
$$

As first example, consider a universe filled with a dark energy fluid with constant $\rho=\Lambda$ and equation of state parameter $\omega=-1$. Assuming that the metric tensor is given by (10), the system (3) has the following solution:

$$
a(t)=a_{0} \exp \left[\sqrt{\frac{k \Lambda}{3}} \frac{\sin (\Omega t)}{\Omega}\right], \quad a_{0} \equiv a(0) .
$$

Note that at the times $t_{n}=(1 / 2+n) \pi / \Omega$ with $n$ integer the scale factor $a(t)$ reaches its maximum (for $n$ even) and minimum (for $n$ odd) values $a_{ \pm} \equiv$ $a_{0} \exp \left[ \pm \sqrt{k \Lambda / 3 \Omega^{2}}\right]$. Also note that, even if the metric (11) is degenerate since $b\left(t_{n}\right)=0$, all physical quantities as the energy density and pressure, the Ricci scalar curvature $R$, etc., are not singular since they can be expressed as functions of the scale factor $a(t)$ (e. g. $R=k[\rho(a)-3 P(a)]$ ), which is finite for all time, see (10). Therefore $t_{n}$ is a fictitious singularity corresponding to the time when the universe passes from an expanding to a contracting epoch and viceversa.

As second example, consider the case of a perfect fluid with equation of state parameter $\omega>-1$. Then the scale factor is 


$$
a(t)=a_{0}\left[1+(1+\omega) \sqrt{\frac{3 k \rho_{0}}{4}} \frac{\sin (\Omega t)}{\Omega}\right]^{\frac{2}{3(1+\omega)}}, \quad a_{0} \equiv a(0) .
$$

The maximum and minimum of the scale factor are now again reached at $t_{n}=$ $(1 / 2+n) \pi / \Omega$ and have the values $a_{ \pm}=a_{0}\left[1 \pm \sqrt{3(1+\omega)^{2} k \rho_{0} / 4 \Omega^{2}}\right]^{\frac{2}{3(1+\omega)}}$. Also in this case $t_{n}$ corresponds to the transition from the expanding to the contracting phases and viceversa and to a fictitious singularity of the metric, since $b\left(t_{n}\right)=0$ but the scale factor $a(t)$ and the relevant physical quantities may remain finite for all time. Indeed for any assignment of the parameter $\Omega$ such that

$$
\Omega>\sqrt{\frac{3 k \rho_{0}}{4}}(1+\omega),
$$

the scale factor $a(t)$ is positive for all time (see (11)) and the big bang singularity of the FRW model is avoided. Hereafter we assume that this restriction, (12), is always enforced.

Of course with a different assignment of $b(t)$ than (9) one obtains different solutions of (3). The simple assignment (9) is an interesting example inasmuch as the scale factor $a(t)$ solution of (3) then mimic the scale factor $\alpha(\tau)$ of the $\Lambda$-CDM model over time intervals much shorter than $T$, see (91). Indeed in this case, as long as $|t| \ll T$ one has $b(t) \simeq 1$ and $\tau \simeq t$ and therefore $a(t) \simeq \alpha(t)$. Since $\Omega$ hence $T$ is arbitrary (see (9) ) one can for instance choose $T$ just a bit smaller than the "standard age" of the universe, say $T \lesssim 1 / H_{0}$ where $H_{0} \simeq 70 \mathrm{Km} / \mathrm{sMpc}$ is the current value of the Hubble parameter.

We stress that the argument based on the transition in the reduced Einstein equations from the time $t$ to the auxiliary variable $\tau$, hence from (3) to (7)illustrated above when the right-hand side is characterized by the simple ideal fluid relation $P=\omega \rho$-remains valid in the more general case in which the right-hand side of (3) corresponds to a universe filled with a mixture of ultra-relativistic and nonrelativistic perfect fluids plus a cosmological constant and an inflaton scalar field; then the solution of (3) and (7) gives, for $|t| \ll T$, the "right" sequence of inflation / radiation domination / matter domination / late time acceleration epochs characterizing the $\Lambda$-CDM universe. While, as discussed above, a suitable assignment of $\Omega$ hence $T$ allows to evade the big bang singularity (see (12) in the case of a perfect fluid with $\omega>-1$ ).

\section{Generalities on isochronous cosmologies}

We complete this paper with some general considerations on these isochronous cosmological models. We have shown how to construct isochronous solutions of (3) from solutions of the FRW equations (7) by considering arbitrary periodic functions $b(t)$ such that their integral $B(t)$ is also periodic and the big bang singularity is 
avoided. In such a way one obtains solutions which are also periodic with the same period of $b(t)$ in the frame $(t, \vec{x})$. Their period is independent of the initial data hence these solutions are isochronous. We call these periodic and singularity free models isochronous cosmologies, to distinguish them from other cyclic models [18.

Let us reemphasize that within any time interval of length appropriately less than $T$ (in particular, less than $T / 2$ for the specific assignment (91)) where the change of the time variable (6) is invertible, the isochronous solutions based on the metric (11) can be mapped into FRW solutions based on the metric (2) by the time reparameterization $t \rightarrow \tau(t)=B(t)$, see (6a). This implies that isochronous solutions are locally (in time) equivalent to FRW solutions in the sense that they give the same physics. However, since the change of time (6) is not a global diffeomorphism, the equivalent FRW frame is not a global system of coordinates, and the equivalence is only local (in time). In fact the isochronous solutions are such that the scale factor $a(t)$ is not a monotonous function of time; moreover the big bang singularity is avoided, a fundamental difference from the FRW cosmology. Isochronous and FRW solutions are locally but not globally equivalent.

From (6) it is easy to recognize that the isochronous solutions $a(t), \rho(t), p(t)$ span only a part of the FRW trajectories $\alpha(\tau), r(\tau), P(\tau)$ and the corresponding isochronous universes consist of an infinite sequence of expansions and contractions. The turning points between each expanding and contracting phase (and vice versa) occur at the times $t_{n}$ when $b(t)$ changes sign and the metric (1) is degenerate since $b\left(t_{n}\right)=0$.

Let us however note that, while the metric (1) is indeed degenerate on the hypersurfaces $t=t_{n}$ since $g_{00}=b\left(t_{n}\right)^{2}=0$ and $g_{\mu \nu}$ is not invertible, these hypersurfaces do not correspond to physical singularities, since $a\left(t_{n}\right)$ neither vanishes nor diverges; hence the energy density $\rho(t)$ and pressure $p(t)$ of the universe are finite at $t=t_{n}$ as well as the Ricci scalar curvature $R=-k[\rho(t)-3 P(t)]$. In fact, by use of the first of (6b) one has

$$
R=\frac{a \ddot{a} b-a \dot{a} \dot{b}+\dot{a}^{2} b}{a^{2} b^{3}}=\frac{\alpha(\tau(t)) \alpha^{\prime \prime}(\tau(t))+\alpha^{\prime 2}}{\alpha(\tau(t))^{2}},
$$

where, above and below, superimposed dots denote as usual differentiations with respect to the time $t$ and appended primes denote differentiations with respect to the argument of the function they are appended to, so that $\alpha^{\prime}(z)=d \alpha(z) / d z$ and $\alpha^{\prime \prime}(z)=d^{2} \alpha(z) / d z^{2}$. One can also compute the Kretschmann invariant $K \equiv$ $R^{\alpha \beta \gamma \theta} R_{\alpha \beta \gamma \theta}$ which turns out to be expressed by the following formulas:

$$
K=\frac{12\left[a^{2} \ddot{a}^{2} b^{2}-2 a^{2} \dot{a} \ddot{a} b \dot{b}+a^{2} \dot{a}^{2} \dot{b}^{2}+\dot{a}^{4} b^{2}\right]}{a^{4} b^{6}}=\frac{12\left[\alpha(\tau(t))^{2} \alpha^{\prime \prime}(\tau(t))+\alpha^{4}\right]}{\alpha(\tau(t))^{4}} .
$$

Therefore both the Ricci scalar and the Kretschmann invariant remain finite for all time $t$. Let us also mention that $R_{i i} \propto \rho(t), R_{00} \propto b(t)^{2} \rho(t)$ and $T_{00} \propto b(t)^{2} \rho(t)$ are also finite at $t_{n}$, while $R^{00} \propto \rho(t) / b(t)^{2}$ and $T^{00} \propto \rho(t) / b(t)^{2}$ are not. Physical singularities are characterized by the fact that scalar quantities such as the energy 
density, the pressure and the Ricci scalar curvature become infinite on the singularity. The fact that these physical scalar quantities remain instead finite at $t=t_{n}$ indicates that the hypersurfaces $t=t_{n}$ are not physical singularities.

Let us further elaborate this point and show that the spacetime with metric (11) is not singular at $t=t_{n}$ and more generally that, with a proper choice of the function $b(t)$, the isochronous solutions are singularity-free, see for instance the case of a perfect fluid with equation of state parameter $\omega$ with the assignment (9) and the condition (12).

Indeed a spacetime is singular if it is not geodesically complete; a spacetime $M$ is geodesically complete if, for every point $q$ of $M$, the $\operatorname{exponential~map~} \exp (q)$ is defined on the entire tangent space $T_{q} M[22$ and geodesics are future- and pastextendible.

Let us suppose that the geodesics associated with the FRW metric (2) are identified by the formula

$$
Y=[\lambda, \vec{Y}(\lambda)],
$$

where the parameter $\lambda$ coincides with the FRW cosmological time $y^{0}=\tau$ along the geodesics. Since the FRW solution has a physical big bang singularity at finite time, say at $\tau=\tau_{s}$, the geodesics (15) are defined for any $\lambda \geq \tau_{s}$ and are pastinextendible to $\lambda<\tau_{s}$, in accordance with the fact that the FRW spacetime is not geodesically complete.

It is easy to show (see Appendix A) that the geodesics associated with the isochronous metric (11) are identified by the analogous formula

$$
X=[\mu, \vec{Y}(B(\mu))]=[\mu, \vec{Y}(\tau(\mu))]
$$

and in this case the parameter $\mu$ coincides with the time $x^{0}=t$ along the geodesics of the isochronous spacetime. Therefore for any function $b(t)$ such that

$$
\tau(t)=B(t)>\tau_{s}, \quad \forall t \in \mathbb{R}
$$

the geodesics associated with the isochronous metric (1) are defined for any $t$ and are past- and future-extendible. These geodesics are open spiraling curves in spacetime, with the space coordinates evolving periodically as functions of the time coordinate $x^{0}=\mu$. It may therefore be concluded that any spacetime associated with the isochronous metric (11) with $b(t)$ fulfilling the condition (17) is geodesically complete and therefore singularity free. This in turn implies that the hypersurfaces $t=t_{n}$ are not physical singularities of the isochronous solutions.

To give an explicit example, we return to the case of a spacetime filled with a perfect fluid with equation of state parameter $\omega>-1 / 3$. The FRW scale factor is then

$$
\alpha(\tau)=a_{0}\left[1+(1+\omega) \sqrt{\frac{3 k \rho_{0}}{4}} \tau\right]^{\frac{2}{3(1+\omega)}}, \quad \alpha_{0} \equiv \alpha(0)
$$


for any $\tau \geq \tau_{s} \equiv-\sqrt{4 / 3 k \rho_{0}} /(1+\omega)$ with the big bang singularity at $\tau_{s}$. Let us consider for instance the FRW light-like geodesics moving along the $Y^{1}$ direction, which are given by

$$
Y=\left[\lambda, Y^{1}(\lambda), Y_{s}^{2}, Y_{s}^{3}\right]
$$

with constant $Y_{s}^{2}$ and $Y_{s}^{3}$ and

$Y^{1}(\lambda)=Y_{s}^{1} \pm \int_{\tau_{s}}^{\lambda} \frac{d \tau^{\prime}}{\alpha\left(\tau^{\prime}\right)}=Y_{s}^{1} \pm \frac{2}{\alpha_{0}(1+3 \omega)} \sqrt{\frac{3}{k \rho_{0}}}\left[1+\frac{3(1+\omega)}{2} \sqrt{\frac{k \rho_{0}}{3}} \lambda\right]^{1-\frac{2}{3(1+\omega)}}$

and are well defined for any $\lambda \geq \tau_{s}$. The corresponding light-like geodesics of the associate isochronous metric will be given by (16) as

$$
X=\left[\mu, X^{1}(\mu), Y_{s}^{2}, Y_{s}^{3}\right]
$$

with

$$
X^{1}(\mu)=Y^{1}(B(\mu))=Y_{s}^{1} \pm \frac{2}{\alpha_{0}(1+3 \omega)} \sqrt{\frac{3}{k \rho_{0}}}\left[1+\frac{3(1+\omega)}{2} \sqrt{\frac{k \rho_{0}}{3}} B(\mu)\right]^{1-\frac{2}{3(1+\omega)}}
$$

and $B(t)$ given by (6). Therefore by assigning $b(t)$ so that (17) is satisfied, e.g. with the assignment (9), the condition (17) reads $\sin (\Omega t) / \Omega>-\sqrt{4 / 3 k \rho_{0}} /(1+\omega)$ yielding the condition (12). Then the geodesics (20) are defined for any $t$, in accordance with the fact that with such a choice of $b(t)$, isochronous metrics are geodesically complete and singularity free, see also (11).

For completeness we also show that the isochronous metric (1) fulfills the junction conditions on the hypersurfaces $t=t_{n}$ and we obtain the expression of the extrinsic curvature on the hypersurfaces $t=$ const. We do so because one might question whether the isochronous metric we have introduced is physically acceptable and, in particular, whether the junction conditions on any hypersurfaces - especially on the hypersurfaces $t=t_{n}$ where the expansion / contraction phases alternate and the isochronous metric is degenerate - are satisfied and the stress-energy tensor is regular everywhere.

Let us consider a hypersurface $\Sigma$ dividing the spacetime in two regions $V^{(+)}$and $V^{(-)}$. The condition that the two metrics $g_{\mu \nu}^{(+)}$and $g_{\mu \nu}^{(-)}$in the two regions $V^{(+)}$and $V^{(-)}$must satisfy in order to join smoothly on $\Sigma$ is that they must be the same on both sides of $\Sigma$ together with their first derivatives, see for instance Eq.(3.7.7) in [20], that is

$$
\left.g_{\mu \nu}^{(+)}\right|_{\Sigma}=\left.g_{\mu \nu}^{(-)}\right|_{\Sigma},\left.\quad g_{\mu \nu, \sigma}^{(+)}\right|_{\Sigma}=\left.g_{\mu \nu, \sigma}^{(-)}\right|_{\Sigma}
$$

From (21) it is therefore evident that the isochronous metric (1) satisfies such junction conditions on the hypersurfaces $t=t_{n}$, where it is infinitely differentiable. We also mention that, if the junction conditions (21) on a hypersurface $\Sigma$ are satisfied, 
the stress energy tensor is regular there and it does not feature a distributional character (thin shells) on $\Sigma$. Therefore the stress-energy tensor associated with the isochronous metric (11) is regular everywhere.

Since the second equation (21) is expressed in terms of the derivatives $g_{\mu \nu, \sigma}$ of the metric tensor, which are not tensors, such a condition is usually expressed in an invariant way by use of the extrinsic curvature, which is diffeomorphism-invariant. Therefore (21) can be recast as

$$
\left.g_{\mu \nu}^{(+)}\right|_{\Sigma}=\left.g_{\mu \nu}^{(-)}\right|_{\Sigma},\left.\quad K_{a b}^{(+)}\right|_{\Sigma}=\left.K_{a b}^{(-)}\right|_{\Sigma}
$$

where $K_{a b}$ is the extrinsic curvature of $\Sigma$ defined as

$$
K_{a b}=n_{\alpha ; \beta} e_{a}^{\alpha} e_{b}^{\beta},
$$

with $n^{\alpha}$ the unitary normal vector to $\Sigma$ and $e_{a}^{\alpha}$ the three unitary tangent vectors to $\Sigma$, so that $g_{\alpha \beta}=n_{\alpha} n_{\beta}-e_{\alpha a} e_{\beta b} \delta^{a b}$, where $n_{\alpha}=g_{\alpha \beta} n^{\beta}$ and $e_{\alpha a}=g_{\alpha \beta} e_{a}^{\beta}$. Note that (21) is more general than (22), since it is valid even where the extrinsic curvature does not exist.

Let us now show that the second equation (22) is also satisfied and the extrinsic curvature is continuous on $t=t_{n}$. The normal and tangent vectors to the hypersurfaces $t=$ const with $t \neq t_{n}$ are $n^{\alpha}=\delta^{\alpha 0} / b(t)$ and $e^{\alpha a}=-\delta^{\alpha a} / a(t)$, so that $n_{\alpha}=b(t) \delta_{\alpha 0}$ and $e_{\alpha a}=a(t) \delta_{\alpha a}$. Furthermore, since $\Gamma_{\alpha \beta}^{0}=\delta_{\alpha \beta} a(t) \dot{a}(t) / b(t)^{2}$ one finds

$$
n_{\alpha ; \beta}=n_{\alpha, \beta}-\Gamma_{\alpha \beta}^{\sigma} n_{\sigma}=\delta_{\alpha 0} \partial_{\beta} b(t)-\Gamma_{\alpha \beta}^{0} n_{0}=\delta_{\alpha 0} \delta_{\beta 0} \dot{b}(t)-\delta_{\alpha \beta} \frac{\dot{a} a}{b} .
$$

Therefore from (23) 24), using (2b) and (6b) which gives $\dot{a}(t)=\alpha^{\prime}(\tau(t)) b(t)$, one has

$$
K_{a b}=-\delta_{a b} \frac{\alpha^{\prime}(\tau[t])}{\alpha(\tau[t])} .
$$

Here, of course, the appended prime denotes differentiation with respect to $\tau$.

This expression implies three facts. Firstly, since the extrinsic curvature is invariant under redefinition of time, it is the same as in the equivalent FRW reference frame (as it should be). Secondly, the extrinsic curvature is only apparently singular on the hypersurfaces $t=t_{n}$. In fact, even though the normal vector $n^{\alpha}(t)=\delta^{\alpha 0} / b(t)$ is not defined at $t=t_{n}$ where $b\left(t_{n}\right)=0$, from (25) and (9) it is clear that both limits of $K_{a b}$ for $t \rightarrow t_{n}^{( \pm)}$exist, are finite and coincide with each other, hence the extrinsic curvature is not singular at $t=t_{n}$. And clearly also the second junction condition in (22) is satisfied at $t=t_{n}$ and both the metric tensor and the stress energy tensor are regular there (and of course elsewhere as well). Thirdly, as long as the inequality (17) holds, the extrinsic curvature (which is proportional to the Hubble parameter of the FRW metric (2)) is finite for any $t$, since, in such a case, $\alpha(\tau(t))>0$ everywhere.

Even though isochronous solutions are singularity-free and possess a regular stress-energy tensor, they are degenerate (non invertible) on the hypersurfaces 
$t=t_{n}$. However, solutions of the Einstein equations featuring degenerate metrics are common in general relativity. In fact the appearance of horizons of events where the metric tensor is degenerate is quite common: for instance the Schwarzschild solution has a metric tensor which is degenerate (and also singular) at its horizon, but it has a well defined physical meaning there. The isochronous solutions introduced in this paper also feature a metric that is degenerate at certain discrete times $t_{n}$, but they are not singular there, indeed they are differentiable and geodesically complete everywhere, therefore they are physically meaningful solutions of the Einstein equations.

A further observation. We have just seen that the isochronous cosmologies introduced in this paper may provide examples of singularity-free Einsteinian spacetimes. Since there are theorems by Hawking and Penrose - based on rather general hypotheses - which guarantee the existence of singularities of the solutions of the Einstein equations 2122, it is reasonable to wonder how the isochronous solutions introduced in this paper manage to be singularity-free. The answer is that these isochronous solutions define spacetimes which are not globally hyperbolic, hence which do not fulfill the hypotheses underpinning the validity of the theorems mentioned above. And they are not globally hyperbolic because they are not stably causal, indeed they do not possess a smooth function of the spacetime coordinates such that its gradient is strictly timelike everywhere [22].

Finally, it is instructive to compare the isochronous metric (1) with the well known Gödel metric [23, providing an exact solution of the Einstein equations in the presence of a cosmological constant and a perfect dust fluid. Just as the isochronous metric, the Gödel metric is geodesically complete hence singularity-free, and not globally hyperbolic. The main difference is that the Gödel metric features closed time-like null curves - this being one of its most remarkable characteristicsand this implies that in this case causality cannot be well defined; while no such phenomenology haunts our isochronous solutions, since all their geodesics are open curves (spiraling in spacetime).

The physical interpretation of the Gödel solution was questioned by Einstein 24]; likewise, the physical interpretation of isochronous solutions based on the metric (11) can be considered a debatable issue. But - just as Einstein did not question the fact that the Gödel solution is a bona fide solution of his field equations [24] there is in our opinion no valid justification to deny that the isochronous metric (1) provides a bona fide solution of Einstein's equations.

\section{Conclusions}

We have shown that for any given FRW cosmology described by the metric (2) it is possible to find an infinite number of isochronous cosmologies described by the periodic metric (10) and that one can always assign the arbitrary function $b(t)$ so that the new metric (1) is singularity free and approximates the FRW metric (2) with 
arbitrary accuracy for an arbitrarily long time (of course, adequately less than the isochrony period $T$, which can be, itself, arbitrarily assigned). One might wonder how general this finding is. To answer this question, we note that the mechanism to find isochronous cosmologies can be applied to any synchronous metric, i.e. to any metric with $g_{00}=1$ and $g_{0 i}=0$. Therefore, as long as one can perform a coordinate transformation that makes a solution of the Einstein equations synchronous, one can find an isochronous solution of these field equations which approximates the original solution for an arbitrarily long time with arbitrary accuracy-indeed, which is locally (but only locally) physically identical to the original solution up to diffeomorphic reparameterization of the time variable. Since it is possible to write most metrics in synchronous form by a diffeomorphic change of coordinates, this makes our finding quite general.

Finally let us repeat (see Remark 1.1) that, while the results that are valid for the rather general (nonrelativistic) many-body problems mentioned at the beginning of this paper can be extended from a classical to a quantal context in a mathematically rigorous manner [1, an analogous quantal extension of the approach introduced in this paper is unfeasible as long as there is no mathematically rigorous theory encompassing general relativity and quantization.

\section{Acknowledgments}

This paper is the outcome of a collaboration among a relativist (FB) and a mathematical physicist (FC) who over the last few years, together with F. Leyvraz, investigated isochronous systems and who was interested in extending some of these findings to a cosmological context. We wish to thank F. Leyvraz for several useful discussions and we regret that he preferred to opt out of co-authorship; and we also like to thank A. Degasperis for enlightening conversations. FB is grateful to G. Calcagni, G. Gonzalez, A. Marciano, A. Melchiorri, F. Mercati, L. Modesto, Y. Rodriguez, M. Saridakis and W. Westra for useful discussions. We also like to thank the Centro Internacional de Ciencias (CIC) in Cuernavaca, Mexico, for the kind hospitality during the Gathering of Scientists on "Integrable systems and the transition to chaos" that took place there in November-December 2012, when this paper was largely drafted. FB thanks the UIS University of Bucaramanga for kind hospitality during the revision of this manuscript. FB is a Marie Curie fellow of the Istituto Nazionale di Alta Matematica Francesco Severi.

\section{Appendix A: Geodesics}

The equation characterizing the geodesics of a spacetime with a given metric $g_{\alpha \beta}$ reads

$$
\frac{d^{2} x^{\beta}}{d \lambda^{2}} g_{\beta \alpha}+\Gamma_{\alpha \beta \gamma} \frac{d x^{\beta}}{d \lambda} \frac{d x^{\gamma}}{d \lambda}=g_{\beta \alpha} \frac{d x^{\beta}}{d \lambda} \frac{1}{L} \frac{d L}{d \lambda},
$$


where Greek indices run from 0 to $3, \Gamma_{\alpha \beta \gamma}=\left(g_{\beta \alpha, \gamma}+g_{\gamma \alpha, \beta}-g_{\beta \gamma, \alpha}\right) / 2$ are the Christoffel symbols, $\lambda$ is a parameter used to parameterize the geodesic and $L=$ $\sqrt{\left(d x^{\alpha} / d \lambda\right)\left(d x^{\beta} / d \lambda\right) g_{\alpha \beta}}$ for timelike geodesics, $L=\sqrt{-\left(d x^{\alpha} / d \lambda\right)\left(d x^{\beta} / d \lambda\right) g_{\alpha \beta}}$ for spacelike geodesics (an analogous, if not quite identical, treatment applies to null geodesics; we omit it to avoid repetition). Note that this equation, (26), is adequate to define the geodesics even where the metric $g_{\alpha \beta}$ is not invertible; it is only the reduction to its more standard version,

$$
\frac{d^{2} x^{\alpha}}{d \lambda^{2}}+\Gamma_{\beta \gamma}^{\alpha} \frac{d x^{\beta}}{d \lambda} \frac{d x^{\gamma}}{d \lambda}=\frac{d x^{\alpha}}{d \lambda} \frac{1}{L} \frac{d L}{d \lambda},
$$

that is not defined when the metric $g_{\alpha \beta}$ is not invertible.

Let us suppose that the geodesics associated with the FRW metric (2) are identified by the formula

$$
Y=\left[Y^{0}(\lambda), \vec{Y}(\lambda)\right]
$$

where $Y^{0}$ coincides with the FRW cosmological time along the geodesics. We use this notation to emphasize the fact that geodesics are geometric curves and they do not depend of their parameterization. Let us specify the geodesic equation (26) (or equivalently (27)) in the case of the FRW metric (2), which gives

$$
\begin{gathered}
\frac{d^{2} Y^{0}}{d \lambda^{2}}+\alpha^{\prime}\left(Y^{0}\right) \alpha\left(Y^{0}\right) \frac{d Y^{i}}{d \lambda} \frac{d Y^{j}}{d \lambda} \delta_{i j}=\frac{d Y^{0}}{d \lambda} \frac{1}{L_{Y}} \frac{d L_{Y}}{d \lambda}, \\
\frac{d^{2} Y^{i}}{d \lambda^{2}}+\frac{\alpha^{\prime}\left(Y^{0}\right)}{\alpha\left(Y^{0}\right)} \frac{d Y^{i}}{d \lambda} \frac{d Y^{0}}{d \lambda}=\frac{d Y^{i}}{d \lambda} \frac{1}{L_{Y}} \frac{d L_{Y}}{d \lambda},
\end{gathered}
$$

where Latin indices run from 1 to $3, \alpha^{\prime}\left(Y^{0}\right) \equiv d \alpha\left(Y^{0}\right) / d Y^{0}$ and $L_{Y}=\sqrt{\left|\left(d Y^{\alpha} / d \lambda\right)\left(d Y^{\beta} / d \lambda\right) g_{\alpha \beta}\right|}$ with $g_{\alpha \beta}$ given by (2) for timelike and spacelike geodesics.

Likewise the geodesics associated with the isochronous metric (11) are given by

$$
X=\left[X^{0}(\lambda), \vec{X}(\lambda)\right]
$$

where again $X^{0}$ coincides with the time along the geodesics of the metric (11). The geodesic equations for the metric (11) are

$$
\begin{gathered}
\frac{d^{2} X^{0}}{d \lambda^{2}}+\frac{b^{\prime}\left(X^{0}\right)}{b\left(X^{0}\right)}\left(\frac{d X^{0}}{d \lambda}\right)^{2}+\frac{a^{\prime}\left(X^{0}\right) a\left(X^{0}\right)}{b^{2}\left(X^{0}\right)} \frac{d X^{i}}{d \lambda} \frac{d X^{j}}{d \lambda} \delta_{i j}=\frac{d X^{0}}{d \lambda} \frac{1}{L_{X}} \frac{d L_{X}}{d \lambda}, \\
\frac{d^{2} X^{i}}{d \lambda^{2}}+\frac{a^{\prime}\left(X^{0}\right)}{a\left(X^{0}\right)} \frac{d X^{i}}{d \lambda} \frac{d X^{0}}{d \lambda}=\frac{d X^{i}}{d \lambda} \frac{1}{L_{X}} \frac{d L_{X}}{d \lambda},
\end{gathered}
$$

where $a^{\prime}\left(X^{0}\right) \equiv d a\left(X^{0}\right) / d X^{0}$ and $L_{X}=\sqrt{\left|\left(d X^{\alpha} / d \lambda\right)\left(d X^{\beta} / d \lambda\right) g_{\alpha \beta}\right|}$ with $g_{\alpha \beta}$ the isochronous metric (1) for timelike and spacelike geodesics. 
Using the fact that $a\left(X^{0}\right) \equiv \alpha\left(B\left(Y^{0}\right)\right)$ (see $\left.6 \mathrm{~b}\right)$ ), it is easy to see that the geodesics (28) and (30), solutions of the systems (29) and (31) respectively, are related by the following equalities

$$
\frac{d Y^{0}(\lambda)}{d \lambda}=b\left(X^{0}(\lambda)\right) \frac{d X^{0}(\lambda)}{d \lambda} ; \quad \frac{d Y^{i}(\lambda)}{d \lambda}=\frac{d X^{i}(\lambda)}{d \lambda} .
$$

These relations can be integrated to give (up to 4 irrelevant integration constants)

$$
Y^{0}(\lambda)=B\left(X^{0}(\lambda)\right) ; \quad Y^{i}(\lambda)=X^{i}(\lambda) .
$$

If the geodesics of the metric (2) are parameterized by $Y^{0}=\lambda=\tau$ and

$$
Y=[\lambda, \vec{Y}(\lambda)]
$$

from (33), using the fact that $X^{i}(\lambda)=Y^{i}\left(Y^{0}=\lambda\right)=Y^{i}\left(Y^{0}=B\left(X^{0}(\lambda)\right)\right)$ and re-parameterizing the geodesic $X$ by use of its proper time $X^{0} \equiv \mu=t$ instead of the parameter $\lambda$, one has that the geodesics of the isochronous metric (1) are given by

$$
X=[\mu, \vec{Y}(B(\mu))]=[t, \vec{Y}(\tau(t))],
$$

which is our final result, see (16) and (17).

For instance, one can easily verify that the curves

$$
X=\left[X^{0}=\lambda, \vec{X}(\lambda)=\vec{X}(0)\right]
$$

are time-like geodesics of the metric (1), solutions of (31a 31b), which correspond to observers at rest in the reference frame of (11). They pass through the hypersurfaces $t=t_{n}$ smoothly, therefore no singularity can be present there.

\section{References}

[1] F. Calogero and F. Leyvraz, General technique to produce isochronous Hamiltonians, J. Phys. A.: Math. Theor. 40, 12931-12944 (2007).

[2] F. Calogero and F. Leyvraz, How to extend any dynamical system so thatit becomes isochronous, asymptotically isochronous or multi-periodic, J. Nonlinear Math. Phys. 16, 311-338 (2009); F. Calogero and F. Leyvraz, Isochronous systems, the arrow of time, and the definition of deterinistic chaos, Lett. Math. Phys. 96, 37-52 (2011). F. Calogero, Isochronous systems, Oxford University Press, 2008 (marginally updated paperback edition, 2012); F. Calogero,Isochronous dynamical system, Phil. Trans. R. Soc. A 369, 1118-1136 (2011).

[3] V. Mukhanov, Physical Foundations of Cosmology, Cambridge University Press, 2005.

[4] E. Komatsu et.al. [WMAP collaboration], Seven-Year Wilkinson Microwave Anisotropy Probe (WMAP) Observations: Cosmological Interpretation, Astrophys. J. Suppl. 192: 18 (2011) arXiv:1001.4538.

[5] B. Jain and A. Taylor,Cross-correlation Tomography: Measuring Dark Energy Evolution with Weak Lensing, Phys. Rev. Lett. 91, 141302 (2003) arXiv:astro-ph/0306046. 
[6] D. J. Eisenstein et al. [SDSS Collaboration], Detection of the Baryon Acoustic Peak in the Large-Scale Correlation Function of SDSS Luminous Red Galaxies, Astrophys. J. 633, 560 (2005) arXiv:astro-ph/0501171.

[7] M. Tegmark et al. [SDSS Collaboration], Cosmological parameters from SDSS and WMAP, Phys. Rev. D 69, 103501 (2004) arXiv:astro-ph/0310723; U. Seljak et al. [SDSS Collaboration], Cosmological parameter analysis including SDSS Ly-alpha forest and galaxy bias: constraints on the primordial spectrum of fluctuations, neutrino mass, and dark energy, Phys. Rev. D 71, 103515 (2005) arXiv:astro-ph/0407372.

[8] S. Perlmutter et al. [SNCP Collaboration], Measurements of Omega and Lambda from 42 High-Redshift Supernovae, Astrophys. J. 517, 565 (1999) arXiv:astro-ph/9812133; A. G. Riess et al. [Supernova Search Team Collaboration], Observational Evidence from Supernovae for an Accelerating Universe and a Cosmological Constant, Astron. J. 116, 1009 (1998) arXiv:astro-ph/9805201.

[9] Planck Collaboration: P. A. R. Adeet al., Planck 2013 results. XVI. Cosmological parameters, arXiv:1303.5076.

[10] S. Capozzielloet al., Cosmographic Constraints and Cosmic Fluids, Galaxies 2013, 1(3), 216-260 (2013) arXiv:1312.1825.

[11] A. Avileset al., Cosmography and constraints on the equation of state of the Universe in various parametrizations, Phys. Rev. D 86, 123516 (2012) arXiv:1204.2007.

[12] E. J. Copelandet al., Dynamics of dark energy, Int. J. Mod. Phys. D 15 :1753-1936 (2006) arXiv:hep-th/0603057.

[13] Bamba et al., Dark energy cosmology: the equivalent description via different theoretical models and cosmography tests, Astrophys. Space Sci. 342: 155-228(2012) arXiv:1205.3421].

[14] P. J. Steinhardt and N. Turok, Cosmic Evolution in a Cyclic Universe, Phys. Rev. D 65, 126003 (2002) arXiv:hep-th/0111098; P. J. Steinhardt and N. Turok, A cyclic model of the universe, Science 296, 1436 (2002); J. Khoury et al., Designing Cyclic Universe Models, Phys. Rev. Lett. 92, 031302 (2004) arXiv:hep-th/0307132; P. J. Steinhardt and N. Turok, Why the cosmological constant is small and positive, Science 312, 1180 (2006) astro-ph/0605173; K. Saaidi et al., Interacting New Agegraphic Dark Energy in a Cyclic Universe, Astrophys. Space Sci. 338, 355 (2012) arXiv:1201.0275; S. Nojiri et al., Cyclic, ekpyrotic and little rip universe in modified gravity, AIP Conf. Proc. 1458, 207 (2011) arXiv:1108.0767]; Y. F. Cai and E. N. Saridakis, Non-singular Cyclic Cosmology without Phantom Menace, J. Cosmol. 17, 7238 (2011) arXiv:1108.6052; V. Sahni and A. Toporensky, Cosmological Hysteresis and the Cyclic Universe, Phys. Rev. D 85, 123542 (2012) arXiv:1203.0395; P. Creminelli and L. Senatore, A smooth bouncing cosmology with scale invariant spectrum, JCAP 0711, 010 (2007) arXiv:hep-th/0702165; Y. S. Piao, Proliferation in Cycle, Phys. Lett. B 677, 1 (2009) arXiv:0901.2644]; J. Zhang et al., Amplification of Curvature Perturbations in Cyclic Cosmology, Phys. Rev. D 82, 123505 (2010) arXiv:1007.2498; Z. G. Liu and Y. S. Piao, Scalar Perturbations Through Cycles, Phys. Rev. D 86, 083510 (2012) arXiv:1201.1371.

[15] J. Khoury et al., The Ekpyrotic Universe: Colliding Branes and the Origin of the Hot Big Bang, Phys. Rev. D 64, 123522 (2001) arXiv:hep-th/0103239; R. Y. Donagi et al., Visible Branes with Negative Tension in Heterotic M-Theory, JHEP 0111, 041 (2011) arXiv:hep-th/0105199; J. Khoury et al., Density Perturbations in the Ekpyrotic Scenario, Phys. Rev. D 66, 046005 (2002) arXiv:hep-th/0109050.

[16] Y. Shtanov and V. Sahni, Bouncing Braneworlds, Phys. Lett. B 557, 1 (2003) arXiv:gr-qc/0208047; T. Biswas et al., Bouncing Universes in String-inspired Gravity, JCAP 0603, 009 (2006) arXiv:hep-th/0508194. 
[17] K. Bamba et al., Periodic Cosmological Evolutions of Equation of State for Dark Energy, Entropy 2012, 14(11), 2351-2374 arXiv:1203.4226.

[18] M. Novello, S.E.P. Bergliaffa, Bouncing Cosmologies, Phys. Rep. 463, 127-213 (2008) arXiv:0802.1634.

[19] See "Cesare Cremonini (philosopher)" in Wikipedia.

[20] E. Poisson, An Advanced Course in General Relativity, University of Guelph (2002) (http://www.physics.uoguelph.ca/ poisson/research/agr.pdf).

[21] C.W. Misner, K.S. Thorne, J.A. Wheeler - Gravitation, Freeman \& Co. (1973).

[22] J. Natario, Relativity and Singularities - A Short Introduction for Mathematicians, Resenhas 6: 309-335 (2005) arXiv:math/0603190.

[23] K. Gödel, An Example of a New Type of Cosmological Solutions of Einstein's Field Equations of Gravitation, Rev. Mod. Phys. 21, 447-450 (1949).

[24] Paul A. Schlipp - Albert Einstein: Philosopher-Scientist, OPEN COURT Publishing Company (1998). 